\title{
Hereditary Angioedema Type I with normal complements
}

\section{Authors:}

- Latifa AlShekaili*1, latifaalshukeili@hotmail.com

- AnasAlazami², AmAlazami@kfshrc.edu.sa

- Sulaiman Al Gazlan', gazlan@kfshrc.edu.sa

- Safiah Al Sumayli ${ }^{1}$, ssumayli@kfshrc.edu.sa

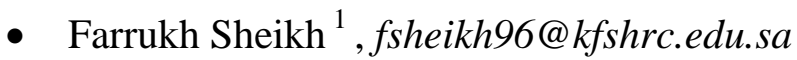

- A.M.KhaliqRehan ${ }^{1,3}$, rehankhaliq@kfshrc.edu.sa

- Rand Arnaout* *1,3, rarnaout@kfshrc.edu.sa (*

(1) King Faisal Specialist Hospital and Research Center, Department of Medicine, Allergy and Immunology section.

(2) King Faisal Specialist Hospital and Research Center, Department of Immunogenetics, MBC03 PO BOX 3354, Riyadh 11211, Saudi Arabia,

(3) Alfaisal University.

None of authors has any potential financial conflict of interest to this manuscript.

\begin{abstract}
Hereditary angioedema (HAE), is an autosomal immunological disease, that is characterized by repetitive diffuse non-pitting painless edema without urticaria or pruritus. HAE is a rare disease with wide range of manifestation, thus diagnosis can be easily missed. Screening for HAE should be warranted in any patient with following manifestations; recurrent self-limiting unexplained abdominal pain, recurrent attacks of angioedema with no clear cause, and personal or family history of asphyxiation due to laryngeal edema in the absence of clear cause.
\end{abstract}

Complement factor 4 level is considered an good screening test for HAE. Where C4 level is very low or depleted by the abnormal $\mathrm{Cl}$ activity especially during acute attack. Special assay for $\mathrm{Cl}$ esterase inhibitor level and activity is available and can indicate if the defect is quantitative or qualitative. However, Cl esterase inhibitor assay cannot exclude HAE type III (due to factor XII mutation), since it will be normal in this case.

In this report, we are describing a family with type I HAE (abnormal Cl esterase inhibitor assay) but with normal C4 level, which indicate C4 level is not the optimum screening test and definitely Cl esterase inhibitor assay should be the standard of care if available for diagnosis of HAE.

\section{Clinical Importance:}

- $\quad$ C4 level is not the optimal for screening test for HAE.

- C1 esterase inhibitor assay should also be the standard of care for diagnosis of HAE type I and II.

- Exploring other possible mechanisms for inhibition of $\mathrm{C} 1$ activity other than that mediated by $\mathrm{C} 1$ esterase inhibitor in certain cases.

\section{Capsule Summary:}

In this report, we are describing a family with type I HAE (abnormal C1 esterase inhibitor assay) but with unusual normal C4 level.

Keywords: Hereditary angioedema, Cl esterase inhibitor ,Complement factor 4.

\section{Abbreviations:}

- HAE : Hereditary angioedema

- C1-esterase INH : C1 esterase inhibitor

- C4: Complement factor 4 


\section{INTRODUCTION}

Hereditary angioedema (HAE), is an autosomal dominant immunological disorder due to heterozygous mutation in the $\mathrm{C} 1$ esterase inhibitor (C1-esterase $\mathrm{INH})$ gene encoded in chromosome 11(1). HAE is characterized by repetitive diffuse non-pitting, painless edema without urticaria or pruritus. It can involve any part of the body including face, extremities, upper respiratory tract and gastrointestinal wall (2). Bradykinin is the key mediator of HAE (3). Three types of HAE have been reported (type I due to low level of $\mathrm{C} 1$ esterase inhibitor, type II due to abnormal function of $\mathrm{C} 1$ esterase inhibitor and type III due to factor XII mutation). HAE could range from minimal swelling of extremities to a life threating asphyxiation due to laryngeal oedema (4). It is a rare disease with a wide range of manifestation, thus diagnosis can be easily missed. Screening for HAE should be warranted in any patient with following manifestations: recurrent self-limiting unexplained abdominal pain, recurrent attacks of angioedema, and personal or family history of asphyxiation due to laryngeal edema in the absence of clear cause.

Complement factor 4 (C4) level is considered a good screening test for HAE (5). Where C4 level is very low or depleted by the abnormal $\mathrm{C} 1$ activity especially during acute attack. Special assay for $\mathrm{C} 1$ esterase inhibitor level and activity is available and can indicate if the defect is quantitative or qualitative. However, C1 esterase inhibitor assay cannot exclude type III HAE (due to factor XII mutation), since it will be normal in this case.

In this report, we are describing a family with type I HAE (abnormal C1 esterase inhibitor assay) but with normal $\mathrm{C} 4$ level, which indicate $\mathrm{C} 4$ level is not the optimal screening test and definitely $\mathrm{C} 1$ esterase inhibitor assay should be the standard of care if available for diagnosis of HAE.

\section{CASE Report (1):}

54 years old gentleman was referred from a general practitioner with long standing(since age of five years ) recurrent abdominal pain and subcutaneous edema involving upper and lower extremities and one attack of facial swelling which was triggered by minor trauma. This patient had eleven years old who suffered from recurrent abdominal pain and eventually died from respiratory asphyxiation.

Before presentation to us, patient was screened with $\mathrm{C} 4$ level during and between the attacks which was always normal. Thus, he was diagnosed as idiopathic angioedema or type III HAE (normal C1-esterase INH). When he was referred to our center for further workup, C1 esterase INH assay was done using radial immunodiffusion, in addition to $\mathrm{C} 2, \mathrm{C} 3$ and $\mathrm{C} 4$ level. $\mathrm{C} 1$ esterase assay was extremely low with normal C4 (Table 1). This was confirmed by repeated testing several times. In addition, Antinuclear antibodies ( ANA), C2, Anti-C1q are normal. The factor 12 was normal which exclude type III HAE. Other causes of angioedema were excluded.

Therefore, he was started on $\mathrm{C} 1$ esterase inhibitor injection 1000 IU during facial attacks or abdominal pain, along with danazole prophylaxis, after which the patient was well controlled

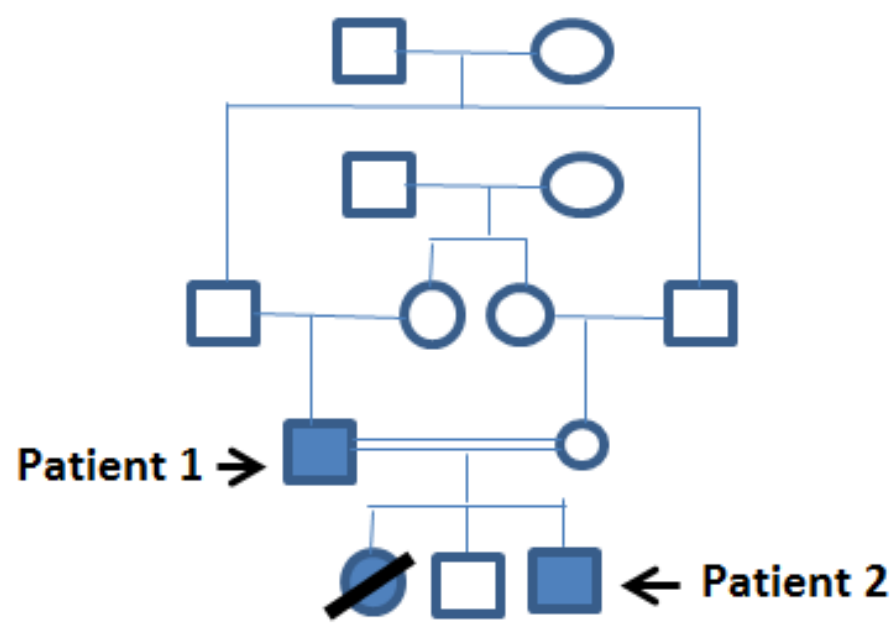

Figure 1. Family Pedigree 


\section{CASE REPORT (2):}

The two years old son of the first patient (Figure 1) started to have similar attacks of abdominal pain and was admitted with respiratory distress due to laryngeal edema for which he required intubation. Thus $\mathrm{C} 1$ esterase inhibitor assay was done and it was abnormal but again with normal $\mathrm{C} 4$ level (Table 1 ). Patient was treated with replacement therapy with $\mathrm{C} 1$ esterase inhibitor. He was improved and discharged.

Table 1. Biochemical Profile

\begin{tabular}{|c|c|c|c|c|}
\hline \multicolumn{2}{|c|}{} & $\begin{array}{c}\text { C4 } \\
\text { g/l }\end{array}$ & C1-INH Antigen & C1-INH Activity \\
\hline \multirow{2}{*}{ Father } & During Attack & $\mathbf{0 . 1 9}$ & $\mathbf{6 . 1 5}$ & $\mathbf{3 3}$ \\
\cline { 2 - 5 } & Between attacks & $\mathbf{0 . 1 6}$ & $\mathbf{5 . 1}$ & $\mathbf{2 8}$ \\
\hline \multirow{3}{*}{ Son } & & & & $\mathbf{6 0}$ \\
\cline { 2 - 5 } & During Attack & $\mathbf{0 . 1 8}$ & $\mathbf{3 . 9 3}$ & $\mathbf{3 4}$ \\
\hline
\end{tabular}

Normal Levels : C4= (0.12 to $0.72 \mathrm{~g} / \mathrm{d}), \mathrm{C} 1-\mathrm{INH}$ Antigen= $19-37 \mathrm{mg} / \mathrm{dl}, \mathrm{C} 1-\mathrm{INH}$ Activity $>67 \%$ normal, $40-67 \%$ equivocal, $<40 \%$ abnormal)

\section{GENETIC FINDINGS}

Whole blood was sampled from both patients, utilized for DNA extraction, then subjected to the Mendeliome assay using a gene panel specific for primary immunodeficiency disorders as described (6). Bioinformatic filtering of the data revealed a previously reported splice site 106 mutation in SERPING1, directly downstream of exon3 (NM_000062.2:c.550+1G>A) (7). Presence of the mutation in both patients was confirmed by Sanger sequencing.

\section{DisCuSSION}

Three types of HAE have been reported; type I and type II due to low quantity or quality of $\mathrm{C} 1$ esterase inhibitor respectively whereas HAE with normal C1-esterase INH level was described as a separate entity which is type III due to a mutation in coagulation factor XII, that causes a gain of function in factor XII. Patients with mutated factor XII have normal level and function of C1 esterase inhibitor $(\mathbf{1 , 8 , 1 0})$, hence, there is no effect on $\mathrm{C} 4$ level. On contrary to factor 12 deficiency, in case of C-esterase abnormalities in function ( $15 \%$ ) or quality ( $85 \%$ ) this will usually leads to excessive proteolysis of $\mathrm{C} 4$ and $\mathrm{C} 2$, since $\mathrm{C} 1$ esterase is the inhibitor of the classical complement, coagulation, fibrinolytic, and kinin-generating pathways $(\mathbf{1 , 8 , 1 0})$. Therefore, usually $\mathrm{C} 4$ level is a good screening test for both type I and II.

However, there was published case report in 2004 describing a patient with type I HAE with low C1esterase INH and normal $\mathrm{C} 4$ without described mutation(11). To our knowledge, our reported family is the second report to have such a profile. Thus, it is highly recommended to emphasize again that screening with $\mathrm{C} 4$ alone may not optimal for the diagnosis of type I and II HAE , in contrary C1estersae INH assay should always measure.

The normal level of C4 in type I HAE sin our patients suggests the presence of another mechanism that inhibit activation of classic complement pathway rather than the classic inhibition by $\mathrm{C} 1$ esterase INH. The possible theory is that such patient could have low production $\mathrm{C} 1$-esterase INH but that small remaining amount of produced $\mathrm{C} 1$-esterase $\mathrm{INH}$ is still capable of $\mathrm{C} 1$ inhibition. One other explanation is that the threshold for inhibition of $\mathrm{C} 1$ activation by $\mathrm{C} 1$ esterase INH enzyme is different from the threshold of activation of kallikrein- kinin system.

There is no previous phenotype-genotype correlation described for $\mathrm{C} 4$ level for the previously reported mutation in the Hungarian study by Kalmar et al (7). Recently published article by Kaplan et al, they described a novel assay to diagnose HAE of all types in which they measure the kallikreinC1-INH complex formation. This test identified $100 \%$ of the patients with types I and II HAE regardless of complement level (12). Therefore, such tests may need to be employed in selected cases.

\section{ACKNOWLEDGMENT}

We thank all immunology staff in king Faisal hospital and research center for their participation. 


\section{REFERENCES}

[1] Longhurst H, Cicardi M. Hereditary angio-oedema. Lancet. 2012;4:474-81. doi: 10.1016/S01406736(11)60935-5.

[2] Davis-Lorton M. An update on the diagnosis and management of hereditary angioedema with abnormal c1 inhibitor, J Drugs Dermatol. 2015 Feb 1;14(2):151-7.

[3] Bouillet L1. Diagnosis of hereditary angioedema, Presse Med. 2015 Jan;44(1):52-6.

[4] Bork K, Hardt J, Schicketanz KH, Ressel N. Clinical studies of sudden upper airway obstruction in patients with hereditary angioedema due to $\mathrm{C} 1$ esterase inhibitor deficiency. Arch Intern Med.2003.

[5] Buyantseva LV, Sardana N, Craig TJ. Update on treatment of hereditary angioedema. Asian Pac J Allergy Immunol. 2012;30:89-98.

[6] Saudi Mendeliome Group. Comprehensive gene panels provide advantages over clinical exome sequencing for Mendelian diseases. Genome Biol. 2015;16:134.

[7] Kalmar L, Bors A, Farkas H, Vas S, Fandl B, Varga L, Füst G, Tordai A. Mutation screening 162 of the $\mathrm{C} 1$ inhibitor gene among Hungarian patients with hereditary angioedema. Hum Mutat. 163 2003;22(6):498.

[8] Morgan BP, Hereditary angioedema--therapies old and new, $N$ Engl J Med. 2010 Aug;363(6):581-3.

[9] Ponard D1, Hereditary angioedema biological diagnosis, Presse Med. 2015 Jan;44(1):57-64.

[10] Kaplan AP, Joseph K, The bradykinin-forming cascade and its role in hereditary angioedema, Ann Allergy Asthma Immunol. 2010;104(3):193.

[11] Y Karim, H Griffiths, and S Deacock, Normal complement C4 values do not exclude hereditary angioedema, J ClinPathol. 2004 Feb; 57(2): 213-214.2

[12] Joseph K1, Bains S, Tholanikunnel BG, Bygum A, Aabom A, Koch C, Farkas H, Varga L, Ghebrehiwet B, Kaplan AP. Novel assay to diagnose hereditary angioedema utilizing inhibition of bradykinin-forming enzymes, Allergy. 2015 Jan;70(1):115-9. 